\title{
COMMENTARY
}

\section{When one cell is enough}

\author{
Rhodri Ceredig*
}

\begin{abstract}
For many years, the hematopoietic stem cell (HSC) has been well characterized in mice as a cell that can singly reconstitute the whole hematopoietic system of primary recipient animals as well as that of secondary hosts. That clinical bone marrow transplantation is a successful treatment strategy is indirect evidence that such a cell exists in humans. To date, similar criteria have not been applied to human HSCs. However, using a humanized mouse model of xenotransplantation, a recent paper shows that single human cells can fully reconstitute the lymphomyeloid system of primary recipient animals and, in some cases, that of secondary hosts.
\end{abstract}

Hematopoiesis, the process whereby blood cells are formed, is a paradigm for how tissue-specific stem cells in this case, hematopoietic stem cells (HSCs) - generate their distinct progeny cell types via partially committed multipotent progenitors (MPPs). Hematopoiesis is one of the few cellular systems in which a tissue-specific stem cell, capable of both self-renewal and complete reconstitution of all lineages, has been demonstrated. However, despite decades of research, there is still considerable debate about exactly how cells end up differentiating along particular lineages [1]. Much of the interest in hematopoiesis emanates from the necessity to understand normal blood cell formation as well as the leukemic process. Studies with mice have unambiguously established that a single genotypically marked HSC can fully reconstitute both the HSC niche and the hematopoietic system of the primary recipient. To show reconstitution, recipient animals must first be purged of endogenous HSCs in order for grafted cells to re-establish hematopoiesis. How the mesenchymal stromal cells, which form an integral part of the HSC niche and which are necessary to support hematopoiesis, survive irradiation is not fully understood. To confirm re-establishment of true HSCs, bone marrow from the primary recipient is then

${ }^{*}$ Correspondence: rhodri.ceredig@nuigalway.ie

Regenerative Medicine Institute, National University of Ireland, University Road, Galway, Ireland transferred to secondary hosts. The kinetics and duration of reconstitution are of critical importance since 'transient' reconstitution does not mean that true HSCs are being analyzed. Magli and colleagues [2] showed that spleen colonies, thought at the time to be derived from HSCs, could appear rapidly and be transient in nature, thereby highlighting the notion that, rather than representing HSCs, the colonies represented the clonal progeny of MPPs. To purify HSCs and unambiguously prove that a single cell can fully reconstitute the hematopoietic compartment, sophisticated tools such as flow cytometry [3], retroviral tagging of injected HSCs [4], and the generation of suitable immune-deficient recipient animals have been developed [5]. All of these experiments with mice raised the question of whether a similar situation was applicable to human HSCs.

Fast forward to 2011 and a paper describing work on human HSCs by Notta and colleagues [6]. This paper, from John Dick's Toronto laboratory, one of the spiritual homes of experimental hematopoiesis, is a truly remarkable technical and scientific tour de force. Experiments addressing the clonality of human HSCs cannot be carried out on humans; therefore, a humanized mouse model must be used. From mouse work, it has become clear that HSCs from genetically different hosts can be recognized by cells, particularly natural killer cells, of the innate immune system. Thus, for the transfer of human HSCs, mice defective in both lymphocyte- and natural killer cell-mediated immunity [5] - in this case, female NSG (nonobese diabetic-scid-IL2Rgc ${ }^{-/}$) recipients must be used. Because they transferred single cells and wanted to maximize the efficiency of reconstitution, the authors, rather than adopt the usual intravenous route of administration, injected purified HSCs directly into the marrow cavity of the long bones of the recipients, a method that is clearly more efficient than the intravenous route. In addition to using elegant flow cytometry to positively select subpopulations of cells with potential HSC activity, the authors took extraordinary care in monitoring how often a single cell was ejected from a syringe into which a single cell had been previously aspirated. This allowed them to apply a correction factor to the observed reconstitution efficiency in vivo.

The authors' long-term strategy is to be able to generate a transcriptome profile of human HSCs in order to characterize the molecular determinants of HSCs for 
stem cell therapy. To achieve this goal, it was imperative to separate genuine long-term reconstituting HSCs from transiently engrafting MPPs. This was achieved by a series of staining and cell-sorting strategies that frequently involved sorting cells twice in order to devise a strategy of separating HSCs from MPPs. HSCs were operationally defined as cells capable of lymphomyeloid reconstitution for at least 20 weeks. Previous experiments had indicated that human HSCs were Thy $1^{+}$; however, Notta and colleagues [6] found distinct activity among Th- $1^{-}$cells, perhaps because of the genotype of recipient mice but also because of the method of reconstitution. A particularly novel aspect of this study was the demonstration that there was a correlation between CD49f expression and HSCs. CD49f is an $\alpha 6$ integrin, a family of molecules that the authors surmised would play a role in HSC niche interactions. Indeed, the authors were able to show that CD49f is a specific HSC marker.

This paper represents a quantum leap in our understanding of human hematopoiesis. Clearly, that more than one phenotypically distinct subpopulation of cells contains operationally defined HSC activity still allows further experimentation in order to resolve differences between such cell types. However, with advances in the sensitivity of the biochemical analytical tools used to dissect the developmental programs of isolated cells, fewer and fewer cells need to be obtained. The ability now to hone in on and purify human HSCs represents a major breakthrough.

\section{Abbreviations}

HSC, hematopoietic stem cell; MPP, multipotent progenitor.

\section{Competing interests}

The author declares that he has no competing interests.

Published: 13 January 2012

\section{References}

1. Ceredig R, Rolink AG, Brown G: Models of haematopoiesis: seeing the wood for the trees. Nat Rev Immunol 2009, 9:293-300.

2. Magli MC, Iscove NN, Odartchenko N: Transient nature of early haematopoietic spleen colonies. Nature 1982, 295:527-529.

3. Spangrude GJ, Heimfeld S, Weissman IL: Purification and characterization of mouse hematopoietic stem cells. Science 1988, 241:58-62.

4. Snodgrass R, Keller G: Clonal fluctuation within the haematopoietic system of mice reconstituted with retrovirus-infected stem cells. EMBO J 1987, 6:3955-3960

5. Shultz LD, Ishikawa F, Greiner DL: Humanized mice in translational biomedical research. Nat Rev Immunol 2007, 7:118-130.

6. Notta F, Doulatov S, Laurenti E, Poeppl A, Jurisica I, Dick JE: Isolation of single human hematopoietic stem cells capable of long-term multilineage engraftment. Science 2011, 333:218-221.

doi:10.1186/scrt92

Cite this article as: Ceredig R: When one cell is enough. Stem Cell Research \& Therapy 2012, 3:1 